\title{
Anomalous dimensions from boson lattice models
}

\author{
Shaun de Carvalho, ${ }^{2, *}$ Robert de Mello Koch, ${ }^{1,2, \dagger}$ and Augustine Larweh Mahu ${ }^{2, *}$ \\ ${ }^{1}$ School of Physics and Telecommunication Engineering, South China Normal University, \\ Guangzhou 510006, China \\ ${ }^{2}$ National Institute for Theoretical Physics, School of Physics and Mandelstam Institute for Theoretical Physics, \\ University of the Witwatersrand, Wits 2050, South Africa
}

(Received 29 April 2018; published 11 June 2018)

\begin{abstract}
Operators dual to strings attached to giant graviton branes in $\operatorname{AdS}_{5} \times S^{5}$ can be described rather explicitly in the dual $\mathcal{N}=4$ super-Yang-Mills theory. They have a bare dimension of order $N$ so that for these operators the large $N$ limit and the planar limit are distinct; summing only the planar diagrams will not capture the large $N$ dynamics. Focusing on the one-loop $S U(3)$ sector of the theory, we consider operators that are a small deformation of a $\frac{1}{2}$-Bogomol'nyi-Prasad-Sommerfield (BPS) multigiant graviton state. The diagonalization of the dilatation operator at one loop has been carried out in previous studies, but explicit formulas for the operators of a good scaling dimension are only known when certain terms which were argued to be small are neglected. In this article, we include the terms which were neglected. The diagonalization is achieved by a novel mapping which replaces the problem of diagonalizing the dilatation operator with a system of bosons hopping on a lattice. The giant gravitons define the sites of this lattice, and the open strings stretching between distinct giant gravitons define the hopping terms of the Hamiltonian. Using the lattice boson model, we argue that the lowest energy giant graviton states are obtained by distributing the momenta carried by the $X$ and $Y$ fields evenly between the giants with the condition that any particular giant carries only $X$ or $Y$ momenta, but not both.
\end{abstract}

DOI: 10.1103/PhysRevD.97.126004

\section{INTRODUCTION}

Motivated by the AdS/CFT correspondence [1-3], there has been dramatic progress in computing the planar spectrum of anomalous dimensions in $\mathcal{N}=4$ superYang-Mills theory. The planar spectrum is now known, in principle, to all orders in the 't Hooft coupling [4]. This has been possible thanks to the discovery of integrability $[5,6]$ in the planar limit of the theory. This spectrum of anomalous dimensions reproduces classical string energies on the $\mathrm{AdS}_{5} \times \mathrm{S}^{5}$ spacetime, in the dual string theory [7].

Much less is known about $\mathcal{N}=4$ super-Yang-Mills theory outside the planar limit. There are many distinct large $N$ but nonplanar limits of the theory that could be considered, and these correspond to a variety of fascinating physical problems. For example, the problem of considering new spacetime geometries (including black hole

542425@students.wits.ac.za

robert@neo.phys.wits.ac.za

*a.larweh@aims.edu.gh

Published by the American Physical Society under the terms of the Creative Commons Attribution 4.0 International license. Further distribution of this work must maintain attribution to the author(s) and the published article's title, journal citation, and DOI. Funded by SCOAP. solutions) corresponds to considering operators with a bare dimension of order $N^{2}$ [8], while giant graviton branes [9-11] are dual to operators with a bare dimension of order $N$. The planar limit does not correctly capture the dynamics of these operators $[12,13]$.

Although much less is known about these large $N$ but nonplanar limits, some progress has been made. Approaches based on group representation theory provide a powerful tool, essentially because they allow us to map the problem of the dynamics of the nonplanar limit-summing the ribbon graphs contributing to correlation functions-into a purely algebraic problem in group theory. Typically, it can be phrased as the construction of a collection of projection operators and their properties. Once the algebraic problem is properly formulated, systematic approaches to it can be developed. As an example of this approach, bases of local gauge invariant operators have been given [14-21]. These bases provide a good starting point from which the anomalous dimensions can be studied. This is basically because they diagonalize the free field two point function and, at weak coupling, operator mixing is highly constrained [22-26]. The resulting operators have a complicated multitrace structure, quite different from the single trace structure relevant for the planar limit and its mapping to an integrable spin chain. The spectrum of anomalous dimensions has been computed for operators that are small deformations of 
1-Bogomol'nyi-Prasad-Sommerfield (BPS) operators. Problems with two distinct characters have been solved; it is possible to simply treat all fields in the operator on the same footing, construct the basis, and then diagonalize [27-30], or alternatively, one can build operators that realize a spacetime geometry or a giant graviton brane and use words constructed from the fields of the conformal field theory (CFT) to describe string excitations $[22,31,32]$. In the approach that treats all fields on the same footing, one simply defines the operators of the basis and considers the diagonalization of the dilatation operator with no physical input from the dual gravity description. When considering states dual to systems of giant gravitons, the Gauss law of the dual giant world volume gauge theory emerges so that in this approach we see open string and membranes are present in the CFT Hilbert space. When using words to describe string excitations, computations in the CFT reproduce the classical values of energies computed in string theory [31,32], the world sheet S-matrix [33], and have led to the discovery of integrable subsectors for string excitations of certain LinLunin-Maldacena (LLM) backgrounds [32]. Clearly, this is a rich problem with hidden simplicity, so further study of these limits is bound to be fruitful. The existence of this hidden simplicity is not unexpected; conventional lore of the large $N$ limit identifies $1 / N$ as the gravitational interaction, so the $N \rightarrow \infty$ limit, in which this interaction is turned off, should be a simple limit.

One next step that can be contemplated is to go beyond small perturbations of the $\frac{1}{2}$-BPS sector. This problem is our main motivation in this study, and we will take a small step in this direction. We will study operators constructed from three complex adjoint scalars $X, Y, Z$ of $\mathcal{N}=4$ superYang-Mills theory. Operators that are a small perturbation of a $\frac{1}{2}$-BPS operator are constructed using mainly $Z$ fields. For these operators, interactions between the $X$ and $Y$ fields are subdominant to interactions between $X$ and $Z$ and between $Y$ and $Z$ fields and can hence be neglected. As we move further from the original $\frac{1}{2}$-BPS operator, more and more $X$ and $Y$ fields are added. At some point, the interactions between the $X$ and $Y$ fields can no longer be neglected. Dealing with these interactions is the focus of our study. We will argue that this is a well-defined problem that can be solved, often explicitly. This is accomplished by phrasing the new $X$ and $Y$ interactions as a lattice model, for essentially free bosons. Thus, we finally end up with a simple problem that is familiar and can be solved. This is the basic achievement of this paper.

Our results show a fascinating structure that deserves to be discussed. The mapping to the lattice model associates a harmonic oscillator to both the $X$ field and to the $Y$ field. Earlier results [29] treating the leading term performed the diagonalization by associating a harmonic oscillator to the $Z$ field, so in the end, we seem to be seeing an equality in the description of the three scalar fields. An even-handed treatment of all three fields is a big step toward being able to treat operators constructed with equal numbers of $X, Y$, and $Z$ fields. This would most certainly go beyond the $\frac{1}{2}$-BPS sector, the main motivation for our study.

In the next section, we review the action of the one-loop dilatation operator $D_{2}$. The action of $D_{2}$ in the $S U(3)$ sector, in the Schur polynomial basis, has been evaluated previously [34], and we simply quote and use the result. We then move to the Gauss graph basis of Ref. [30], in which the terms in $D_{2}$ arising from $Z$ and $Y$ or $Z$ and $X$ interactions are diagonal. Again, this is a known result, and we simply use it. The Gauss graph basis has a natural interpretation in terms of giant graviton branes and their open string excitations. We will often use this language of branes and strings. We then come to the central term of interest: the term in $D_{2}$ arising from $X$ and $Y$ interactions. Denote this term by $D_{2}^{X Y}$. We will carefully evaluate this term, arriving at a rather simple formula, which is the starting point for Sec. III. The explicit expression for $D_{2}^{X Y}$ can easily be identified with a lattice model for a collection of bosons. The giant gravitons define the sites of this lattice, and the open string excitations determine the lattice Hamiltonian. Section IV diagonalizes the dilatation operator for a number of giants plus open string configurations, arriving at detailed and explicit expressions for both the anomalous dimensions and for the operators of a definite scaling dimension. Our conclusions and some discussion are given in Sec. V.

\section{ACTION OF THE ONE-LOOP DILATATION OPERATOR}

We combine the six Hermitian adjoint scalars of $\mathcal{N}=4$ super-Yang-Mills theory into three complex combinations, denoted $X, Y$, and $Z$. The operators we consider are constructed using $n Z$ 's, $m Y$ 's, and $p \quad X$ 's. Operators that are dual to giant graviton branes are constructed using $n+m+p \sim N$ fields. We will focus on operators that are small deformations of $\frac{1}{2}$-BPS operators, achieved by choosing $n \gg m+p$. We will fix $\frac{m}{p} \sim 1$ as $N \rightarrow \infty$ and treat $\frac{m}{n}$ as a small parameter. The collection of operators constructed using $X, Y$, and $Z$ fields are often referred to as the $S U(3)$ sector. This is not strictly speaking correct since these operators do mix with operators containing fermions. At one loop, however, this is a closed sector.

Our starting point is the action of the one-loop dilatation operator of the $S U(3)$ sector

$$
D_{2}=D_{2}^{Y Z}+D_{2}^{X Z}+D_{2}^{X Y},
$$

where

$$
D_{2}^{A B} \equiv g_{\mathrm{YM}}^{2} \operatorname{Tr}\left([A, B]\left[\partial_{A}, \partial_{B}\right]\right)
$$

on the restricted Schur polynomial basis. This has been evaluated in Ref. [34]. Further, the terms $D_{2}^{Y Z}$ and $D_{2}^{X Z}$ have been diagonalized. The operators of a definite scaling 
dimension $O_{R, r}(\sigma)$, called Gauss graph operators [28,30], are labeled by a pair of Young diagrams $R \vdash n+m+p$ and $r \vdash n$ as well as a permutation $\sigma \in S_{m} \times S_{p}$. Although these labels arise when diagonalizing $D_{2}^{Y Z}$ and $D_{2}^{X Z}$ in the CFT, they have a natural interpretation in the dual gravitational description in terms of giant graviton branes plus open string excitations. A Young diagram $R$ that has $q$ rows corresponds to a system of $q$ giant gravitons. The $Y$ and $X$ fields describe the open string excitations of these giants, so there are $m+p$ open strings in total. We can describe the state of the system using a graph, with nodes of the graph representing the branes (and hence rows of $R$ ) and directed edges of the graph describing the open string excitations (represented by $X$ and $Y$ fields in the CFT). Each directed edge ends on any two (not necessarily distinct) of the $q$ branes. The only configurations that appear when $D_{2}^{Y Z}$ and $D_{2}^{X Z}$ are diagonalized have the same number of strings starting or terminating on any given giant, for the $X$ and $Y$ strings separately $[30,34]$. Thus, the Gauss law of the brane world volume theory implied by the fact that the giant graviton has a compact world volume [35] emerges rather naturally in the CFT description. Since every terminating edge end point can be associated to a unique emanating end point, we can give a nice description of how the open strings are connected to the giants by specifying how the terminating and emanating end points are associated. The permutation $\sigma \in S_{m} \times S_{p}$ describes how the $m Y$ 's and the $p X$ 's are draped between the $q$ giant gravitons by describing this association [30,34]. The explicit form of the Gauss graph operators is $[30,34]$

$$
\begin{aligned}
O_{R, r}^{\vec{m}, \vec{p}}(\sigma)= & \frac{\left|H_{X} \times H_{Y}\right|}{\sqrt{p ! m !}} \sum_{j, k} \sum_{s \vdash m} \sum_{t \vdash p} \sum_{\vec{\mu}_{1}, \vec{\mu}_{2}} \sqrt{d_{s} d_{t}} \Gamma_{j k}^{(s, t)}(\sigma) \\
& \times B_{j \vec{\mu}_{1}}^{(s, t) \rightarrow 1_{H_{X} \times H_{Y}}} B_{k \vec{\mu}_{2}}^{(s, t) \rightarrow 1_{H_{X} \times H_{Y}}} O_{R,(t, s, r) \vec{\mu}_{1} \vec{\mu}_{2}} .
\end{aligned}
$$

Each box in $R$ is associated with one of the complex fields. $r$ is a label for the $Z$ fields. The graph $\sigma$ encodes important information. The number of $Y$ (or $X$ ) strings terminating on the $i$ th node, which equals the number of $Y$ (or $X$ ) strings emanating from the $i$ th node, is denoted by $m_{i}$ (or $p_{i}$ ). $m_{i}$ (or $p_{i}$ ) also counts the number of boxes in the $i$ th row of $R$ that correspond to $Y$ (or $X$ ) fields. We will often assemble $m_{i}$ and $p_{i}$ into the vectors $\vec{m}$ and $\vec{p}$. The number of $Y$ (or $X$ ) strings stretching between nodes $i$ and $k$ is denoted $m_{i k}$ (or $p_{i k}$ ), while the number of strings stretching from node $i$ to node $k$ is denoted $m_{i \rightarrow k}\left(\right.$ or $p_{i \rightarrow k}$ ). A Young diagram with $k$ boxes $a \vdash k$ labels an irreducible representation of $S_{k}$ with dimension $d_{a}$. The branching coefficients $B_{j \vec{\mu}_{1}}^{(s, t) \rightarrow 1_{H_{X} \times H_{Y}}}$ resolve the operator that projects from $(s, t)$, with $s \vdash m, t \vdash p$, an irreducible representation of $S_{m} \times S_{p}$, to the trivial (identity) representation of the product group $H_{Y} \times H_{X}$ with $H_{Y}=S_{m_{1}} \times S_{m_{2}} \times \cdots S_{m_{q}}$ and $H_{X}=S_{p_{1}} \times S_{p_{2}} \times \cdots S_{p_{q}}$, i.e.,
$\frac{1}{H_{X} \times H_{Y}} \sum_{\gamma \in H_{X} \times H_{Y}} \Gamma_{i k}^{(s, t)}(\gamma)=\sum_{\vec{\mu}} B_{i \vec{\mu}}^{(s, t) \rightarrow 1_{H_{X} \times H_{Y}}} B_{k \vec{\mu}}^{(s, t) \rightarrow 1_{H_{X} \times H_{Y}}}$.

$\Gamma_{j k}^{(s, t)}(\sigma)$ is a matrix (with row and column indices $j k$ ) representing $\sigma \in S_{m} \times S_{p}$ in irreducible representation

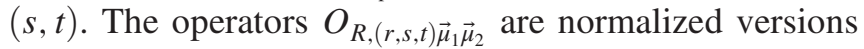
of the restricted Schur polynomials [18]

$$
\begin{aligned}
\chi_{R,(t, s, r) \vec{\mu}_{1} \vec{\mu}_{2}}(Z, Y, X)= & \frac{1}{n ! m ! p !} \sum_{\sigma \in S_{n+m+p}} \chi_{R,(t, s, r) \vec{\mu}_{1} \vec{\mu}_{2}}(\sigma) \\
& \times \operatorname{Tr}\left(\sigma Z^{\otimes n} Y^{\otimes m} X^{\otimes p}\right),
\end{aligned}
$$

which themselves provide a basis for the gauge invariant operators of the theory. The restricted characters $\chi_{R,(t, s, r) \vec{\mu}_{1} \vec{\mu}_{2}}(\sigma)$ are defined by tracing the matrix representing group element $\sigma$ in representation $R$ over the subspace giving an irreducible representation $(r, s, t)$ of the $S_{n} \times$ $S_{m} \times S_{p}$ subgroup. There is more than one choice for this subspace, and the multiplicity labels $\vec{\mu}_{1} \vec{\mu}_{2}$ resolve this ambiguity, for the row and column index of the trace. The operators $O_{R,(t, s, r) \vec{\mu}_{1} \vec{\mu}_{2}}$ given by

$O_{R,(t, s, r) \vec{\mu}_{1} \vec{\mu}_{2}}=\sqrt{\frac{\text { hooks }_{r} \text { hooks }_{s} \text { hooks }_{t}}{\operatorname{hooks}_{R} f_{R}}} \chi_{R,(t, s, r) \vec{\mu}_{1} \vec{\mu}_{2}}$

have unit two point function. hooks ${ }_{r}$ stands for the product of hook lengths of Young diagram $r$, and $f_{R}$ stands for the product of the factors of Young diagram $R$.

The above construction is rather technical, so it is useful to describe some simple examples. A simple example which illustrates the above formulas is provided by taking $R=\square, r=\boxminus, s=\square$, and $t=\square$. Notice that $(r, s, t)$ is a one-dimensional irreducible representation of $S_{2} \times S_{1} \times S_{1}$. In this case, $(r, s, t)$ is obtained once after restricting to the subgroup, so we do not need any multiplicity labels. The representation $R$ is three dimensional, and a basis for the vector space carrying the representation can be labeled using the three standard tableau
$|1\rangle=\left|\begin{array}{|l|l|l}\hline & 3 & 2 \\ \hline 1 & \end{array}\right\rangle$
$\left.|2\rangle=\begin{array}{|l|l|l}\hline 4 & 3 & 1 \\ \hline 2 & & \end{array}\right\rangle$
$|3\rangle=$\begin{tabular}{|l|l|l}
\hline 4 & 2 & 1 \\
\hline 3 &
\end{tabular}.

The state $|3\rangle$ is the $(r, s, t)$ subspace, so the restricted character is

$$
\chi_{\square \square,(\square, \square, \square)}(\sigma)=\left\langle 3\left|\Gamma_{\square} \square(\sigma)\right| 3\right\rangle .
$$

Using this restricted character, we find 


$$
\begin{aligned}
\chi_{\square \square,(\square, \square, \square)}(Z, Y, X)= & \frac{1}{2}\left[\operatorname{Tr}(Z)^{2} \operatorname{Tr}(Y) \operatorname{Tr}(X)-\operatorname{Tr}\left(Z^{2}\right) \operatorname{Tr}(Y) \operatorname{Tr}(X)\right. \\
& +\operatorname{Tr}(Z Y) \operatorname{Tr}(Z) \operatorname{Tr}(X)+\operatorname{Tr}(Z X) \operatorname{Tr}(Z) \operatorname{Tr}(Y)+\operatorname{Tr}(Z)^{2} \operatorname{Tr}(Y X) \\
& -\operatorname{Tr}\left(Z^{2} Y\right) \operatorname{Tr}(X)-\operatorname{Tr}\left(Z^{2} X\right) \operatorname{Tr}(Y)+\operatorname{Tr}(Z Y X) \operatorname{Tr}(Z) \\
& \left.+\operatorname{Tr}(Z X Y) \operatorname{Tr}(Z)-\operatorname{Tr}\left(Z^{2}\right) \operatorname{Tr}(Y X)-\operatorname{Tr}\left(Z^{2} Y X\right)-\operatorname{Tr}\left(Z^{2} X Y\right)\right],
\end{aligned}
$$

Thus, we see that the restricted Schur polynomials are sums over all possible traces structures with coefficients set by the restricted characters. To really trust our approximations and the brane interpretation, we would need to have many (order $N$ ) Z's in each row of $r$. However, our goal is simply to illustrate the construction in a simple setting. With this in mind, we will abuse the language and refer to each row of $r$ as a giant graviton. Since we have two rows in $r$, there are two giants. There are two open strings (represented by the $X$ and $Y$ fields), and in this simple example, the restricted Schur operator and Gauss graph operators are identical. This is the case whenever we have a single $X$ and a single $Y$ field. In the generic situation, Eq. (2.3) says we must sum over all possible irreducible representations of the impurities, with the coefficient set by a permutation $\sigma$. To translate permutation $\sigma \in S_{m} \times S_{p}$ into an open string configuration, label the fields $X$ and $Y$ from 1 to $p$ for the $X$ 's and $p+1$ to $p+m$ for the $Y$ 's. Each row of $r$ is a giant graviton. The numbers $\left(p_{1}, p_{2}, \ldots, p_{q}\right)$ tell us how the $X$ strings [and $\left(m_{1}, m_{2}, \ldots, m_{q}\right)$ tell us how the $Y$ strings] are distributed among the giants. The permutation $\sigma$ then tells us how to join the strings. So, for

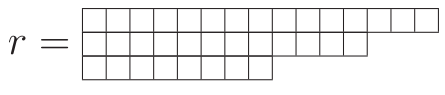

and $\vec{p}=(1,1,1), \vec{m}=(1,1,0)$, and $\sigma=(123)(45)$, we would have the open string plus giant configuration shown in Fig. 1.

The action of the dilatation operator on the Gauss graph operators is $[28,30,34]$

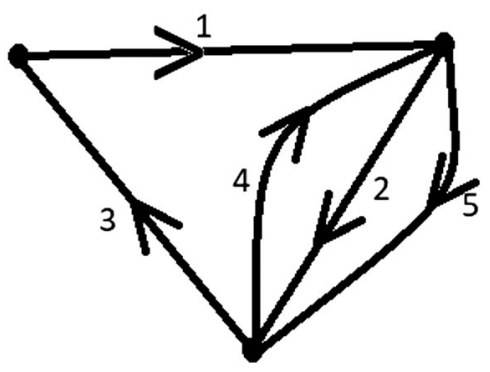

FIG. 1. An example of a Gauss graph for a Young diagram $r$ with three rows, $\vec{p}=(1,1,1), \vec{m}=(1,1,0)$, and $\sigma=(123)(45)$. Strings labeled 1,2 , or 3 are $X$ 's. Strings labeled 4 or 5 are $Y$ 's.

$$
\begin{aligned}
& D_{2}^{Y Z} O_{R, r}^{\vec{m}, \vec{p}}(\sigma)=-g_{\mathrm{YM}}^{2} \sum_{i<j} m_{i j}(\sigma) \Delta_{i j} O_{R, r}^{\vec{m}, \vec{p}}(\sigma) \\
& D_{2}^{X Z} O_{R, r}^{\vec{m}, \vec{p}}(\sigma)=-g_{\mathrm{YM}}^{2} \sum_{i<j} p_{i j}(\sigma) \Delta_{i j} O_{R, r}^{\vec{m}, \vec{p}}(\sigma),
\end{aligned}
$$

where $\Delta_{i j}=\Delta_{i j}^{-}+\Delta_{i j}^{0}+\Delta_{i j}^{+}$[29]. We will now spell out the action of the operators $\Delta_{i j}^{+}, \Delta_{i j}^{0}$, and $\Delta_{i j}^{-}$. Denote the row lengths of $r$ by $l_{r_{i}}$. The Young diagram $r_{i j}^{+}$is obtained by deleting a box from row $j$ and adding it to row $i$. The Young diagram $r_{i j}^{-}$is obtained by deleting a box from row $i$ and adding it to row $j$. In terms of these Young diagrams, we have

$$
\begin{aligned}
& \Delta_{i j}^{0} O_{R, r}^{\vec{m}, \vec{p}}(\sigma)=-\left(2 N+l_{r_{i}}+l_{r_{j}}\right) O_{R, r}^{\vec{m}, \vec{p}}(\sigma) \\
& \Delta_{i j}^{+} O_{R, r}^{\vec{m}, \vec{p}}(\sigma)=\sqrt{\left(N+l_{r_{i}}\right)\left(N+l_{r_{j}}\right)} O_{R_{i j}^{\vec{m}}, \vec{p}}^{\vec{p}}, r_{i j}^{+} \\
& \Delta_{i j}^{-} O_{R, r}^{\vec{m}, \vec{p}}(\sigma)=\sqrt{\left(N+l_{r_{i}}\right)\left(N+l_{r_{j}}\right)} O_{R_{i j}^{-}, \vec{p}, r_{i j}^{-}}^{\vec{m}}(\sigma) .
\end{aligned}
$$

Notice that $D_{2}^{Y Z}$ and $D_{2}^{X Z}$ in (2.10) are not yet diagonal; they still mix operators with different $R, r$ labels. This last diagonalization, however, is rather simple; it maps into diagonalizing a collection of decoupled oscillators as demonstrated in Ref. [29]. We will call these $Z$ oscillators, since they are associated to the $r$ label which organizes the $Z$ fields. It is clear that $D_{2}^{X Y}$ does not act on the $r$ label so that, in the end, the contribution from $D_{2}^{X Y}$ simply shifts the ground state eigenvalue of the $Z$ oscillators.

We will now focus on the term $D_{2}^{X Y}$. Recall that our operators are built with many more $Z$ fields than $X$ or $Y$ fields $(n \gg p+m)$. Since this term contains no derivatives with respect to $Z$, it is subleading (of order $\frac{m}{n}$ ) when compared to $D_{2}^{Y Z}$ and $D_{2}^{X Z}$. Diagonalizing this operator is the main goal of this article, so it is useful to sketch the derivation of the matrix elements of $D_{2}^{X Y}$ in the Gauss graph basis. We will simply quote existing results that we need, giving complete details only for the final stages of the evaluation, which are novel. The reader will find useful background material in Ref. [34]. The action of this term on the restricted Schur polynomial basis was computed in Ref. [34]. The result is 


$$
\begin{aligned}
D_{2}^{X Y} O_{R,(t, s, r) \vec{\mu} \vec{\nu}}= & \sum_{R^{\prime}} \sum_{T,(y, x, w) \vec{\alpha} \vec{\beta}} \mathcal{C} \operatorname{Tr}_{R \oplus T}\left(\left[P_{1}, \Gamma^{R}(1, p+1)\right]\right. \\
& \left.\times I_{R^{\prime} T^{\prime}}\left[P_{2}, \Gamma^{T}(1, p+1)\right] I_{T^{\prime}, R^{\prime}}\right) O_{T,(y, x, w) \vec{\beta} \vec{\alpha}},
\end{aligned}
$$

where

$$
\begin{aligned}
& \mathcal{C}=-g_{\mathrm{YM}}^{2} c_{R R^{\prime}} \frac{d_{T} m p}{d_{x} d_{y} d_{w}(n+m+p) d_{R^{\prime}}} \\
& \times \sqrt{\frac{f_{T} \text { hooks }_{T} \text { hooks }_{r} \text { hooks }_{s} \text { hooks }_{t}}{f_{R} \text { hooks }_{R} \text { hooks }_{w} \text { hooks }_{x} \text { hooks }_{y}}} \\
& P_{1}=P_{R,(t, s, r) \vec{\mu} \vec{\nu}} \quad P_{2}=P_{T,(y, x, w) \vec{\alpha} \vec{\beta}} .
\end{aligned}
$$

$\Gamma^{S}(\sigma)$ is the matrix representing $\sigma \in S_{n+m+p}$ in irreducible representation $S \vdash n+m+p$. Young diagram $R^{\prime}$ is obtained from Young diagram $R$ by dropping a single box, with $c_{R R^{\prime}}$ denoting the factor of this box. $I_{T^{\prime} R^{\prime}}, I_{R^{\prime} T^{\prime}}$, $P_{1}$, and $P_{2}$ are intertwining maps. $I_{T^{\prime} R^{\prime}}$ maps from the carrier space of $R^{\prime}$ to the carrier space of $T^{\prime}$. It is only nonvanishing if $T^{\prime}$ and $R^{\prime}$ are equal as Young diagrams implying that operators labeled by $R$ and $T$ can only mix if they differ by the placement of a single box. The operators
$P_{1}$ and $P_{2}$ are the intertwining maps used in the construction of the restricted Schur polynomials. It is challenging to evaluate the above expression explicitly, basically because it is difficult to construct $P_{1}$ and $P_{2}$. However, the above expression has not yet employed the simplifications of large $N$. To do this, following Ref. [28], we will use the displaced corners approximation. This approximation assumes that the difference of the number of boxes in any two rows of $R$ is of order $N$. In this situation, the action of the $S_{m} \times S_{p}$ subgroup simplifies so much that the relevant restricted characters can be computed and a complete explicit characterization of the multiplicity labels on the restricted Schur polynomials is possible. The corrections to the displaced corners approximation are suppressed by the inverse of the difference in length of rows of $R$. After applying the approximation, we obtain [34]

$$
D_{2}^{X Y} O_{R,(t, s, r) \vec{\mu} \vec{\nu}}=\sum_{T,(w, v, u) \vec{\alpha} \vec{\beta}} \tilde{M}_{R,(t, s, r) \vec{\mu} \vec{\nu} T,(w, v, u) \vec{\alpha} \vec{\beta}} O_{T,(w, v, u) \vec{\alpha} \vec{\beta}},
$$

where

$$
\begin{aligned}
\tilde{M}_{R,(t, s, r) \vec{\mu} \vec{\nu} T,(w, v, u) \vec{\alpha} \vec{\beta}}= & -g_{\mathrm{YM}}^{2} \sum_{R^{\prime}} \delta_{R_{i}^{\prime} T_{k}^{\prime}} \delta_{r u} \frac{p m}{\sqrt{d_{s} d_{t} d_{w} d_{v}}} \sqrt{\frac{c_{R R^{\prime}} c_{T T^{\prime}}}{l_{R_{i}} l_{T_{k}}}} \operatorname{Tr}\left[E_{k i}^{(1)} P_{t \alpha_{1} \beta_{1} ; s \alpha_{2} \beta_{2}}^{(\vec{p}, \vec{m})} E_{i k}^{(p+1)} P_{w \mu_{1} \nu_{1} ; v \mu_{2} \nu_{2}}^{\left(\vec{p}^{\prime}, \vec{m}^{\prime}\right)}\right. \\
& -E_{c i}^{(1)} E_{k c}^{(p+1)} P_{t \alpha_{1} \beta_{1} ; s \alpha_{2} \beta_{2}}^{(\vec{p}, \vec{m})} E_{a k}^{(1)} E_{i a}^{(p+1)} P_{w \mu_{1} \nu_{1} ; v \mu_{2} \nu_{2}}^{\left(\vec{p}^{\prime}, \vec{m}^{\prime}\right)}-E_{k c}^{(1)} E_{c i}^{(p+1)} P_{t \alpha_{1} \beta_{1} ; s \alpha_{2} \beta_{2}}^{(\vec{p}, \vec{m})} E_{i a}^{(1)} E_{a k}^{(p+1)} P_{w \mu_{1} \nu_{1} ; \mu_{2} \nu_{2}}^{\left(\vec{p}^{\prime}, \vec{m}^{\prime}\right)} \\
& \left.+E_{k i}^{(p+1)} P_{t \alpha_{1} \beta_{1} ; s \alpha_{2} \beta_{2}}^{(\vec{p}, \vec{m})} E_{i k}^{(1)} P_{w \mu_{1} \nu_{1} ; v \mu_{2} \nu_{2}}^{\left(\vec{p}^{\prime}, \vec{m}^{\prime}\right)}\right] .
\end{aligned}
$$

The trace in this expression is over the tensor product $V_{q}^{\otimes n+m}$, where $V_{q}$ is the fundamental representation of $U(q)$. The intertwining maps used to define the restricted Schur polynomials ( $P_{1}$ and $P_{2}$ above) factor into an action on the boxes associated to the $Z$ fields, an action on the boxes associated to the $Y$ fields, and an action on the boxes associated to the $\mathrm{X}$ fields. The intertwining maps ${ }^{1} P_{t \alpha_{1} \beta_{1} ; s \alpha_{2} \beta_{2}}^{(\vec{p}, \vec{m})}$ and $P_{w \mu_{1} \nu_{1} ; v \mu_{2} \nu_{2}}^{\left(\vec{p}^{\prime}, \vec{m}^{\prime}\right)}$ are the actions of the intertwining maps on the $X$ and $Y$ fields only. This happens because the trace over the $Z$ field indices, which is simple as the dilatation operator $D_{2}^{X Y}$ does not act on the $Z$ fields, has been performed. Young diagram $R_{i}^{\prime}$ is obtained from $R$ by dropping a single box from row $i$, and $T_{k}^{\prime}$ is obtained from $T$ by dropping a single box from row $k$.

\footnotetext{
${ }^{1}$ A very explicit algorithm for the construction of these maps has been given in Ref. [28].
}

The result (2.16) gives the $D_{2}^{X Y}$ term in the dilatation operator, as a matrix that must be diagonalized. As we will see, all three terms in $D_{2}$ are simultaneously diagonalizable at large $N$, so it is convenient to employ the Gauss graph basis which already diagonalizes both $D_{2}^{Z Y}$ and $D_{2}^{Z X}$. The problem of diagonalizing $D_{2}^{X Y}$ then amounts to a diagonalization on degenerate subspaces of $D_{2}^{Z Y}$ and $D_{2}^{Z X}$. Thus, the original diagonalization of an enormous matrix is replaced by diagonalizing a number of smaller matrices-a significant simplification. Applying the results of Ref. [34], we find that, after the change in basis,

$$
D_{2}^{X Y} \hat{O}_{R, r}^{\vec{m}, \vec{p}}\left(\sigma_{1}\right)=M_{R, r, \sigma_{1} T, t, \sigma_{2}}^{\vec{m}, \vec{O}} \hat{O}_{T, t}^{\vec{m}, \vec{p}}\left(\sigma_{2}\right),
$$

where 


$$
\begin{aligned}
M_{R, r, \sigma_{1} T, t, \sigma_{2}}^{\vec{m}, \vec{p}}= & -g_{\mathrm{YM}}^{2} \frac{1}{\sqrt{\left|O_{R, r}^{\vec{m}, \vec{p}}\left(\sigma_{1}\right)\right|^{2}\left|O_{T, t}^{\vec{m}, \vec{p}}\left(\sigma_{2}\right)\right|^{2}}} \sum_{R^{\prime}} \frac{\delta_{R_{i}^{\prime} T_{k}^{\prime}} \delta_{r u}}{(p-1) !(m-1) !} \sqrt{\frac{c_{R R^{\prime}} c_{T T^{\prime}}}{l_{R_{i}} l_{T_{k}}} \sum_{\psi_{1} \in S_{\vec{p}} \times S_{\vec{m}}} \sum_{\psi_{2} \in S_{\vec{p}^{\prime}} \times S_{\vec{m}^{\prime}}}} \\
& \times\left[\left\langle\vec{p}^{\prime}, \vec{m}^{\prime}\left|\sigma_{2} \psi_{2}^{-1} E_{k i}^{(1)} \psi_{1}\right| \vec{p}, \vec{m}\right\rangle\left\langle\vec{p}, \vec{m}\left|\sigma_{1}^{-1} \psi_{1}^{-1} E_{i k}^{(p+1)} \psi_{2}\right| \vec{p}^{\prime}, \vec{m}^{\prime}\right\rangle\right. \\
& -\left\langle\vec{p}^{\prime}, \vec{m}^{\prime}\left|\sigma_{2} \psi_{2}^{-1} E_{c i}^{(1)} E_{k c}^{(p+1)} \psi_{1}\right| \vec{p}, \vec{m}\right\rangle\left\langle\vec{p}, \vec{m}\left|\sigma_{1}^{-1} \psi_{1}^{-1} E_{a k}^{(1)} E_{i a}^{(p+1)} \psi_{2}\right| \vec{p}^{\prime}, \vec{m}^{\prime}\right\rangle \\
& -\left\langle\vec{p}^{\prime}, \vec{m}^{\prime}\left|\sigma_{2} \psi_{2}^{-1} E_{k c}^{(1)} E_{c i}^{(p+1)} \psi_{1}\right| \vec{p}, \vec{m}\right\rangle\left\langle\vec{p}, \vec{m}\left|\sigma_{1}^{-1} \psi_{1}^{-1} E_{i a}^{(1)} E_{a k}^{(p+1)} \psi_{2}\right| \vec{p}^{\prime}, \vec{m}^{\prime}\right\rangle \\
& \left.+\left\langle\vec{p}^{\prime}, \vec{m}^{\prime}\left|\sigma_{2} \psi_{2}^{-1} E_{k i}^{(p+1)} \psi_{1}\right| \vec{p}, \vec{m}\right\rangle\left\langle\vec{p}, \vec{m}\left|\sigma_{1}^{-1} \psi_{1}^{-1} E_{i k}^{(1)} \psi_{2}\right| \vec{p}^{\prime}, \vec{m}^{\prime}\right\rangle\right] .
\end{aligned}
$$

Here, the Gauss graph operators $\hat{O}_{R, r}^{\vec{m}, \vec{p}}\left(\sigma_{1}\right)$ are normalized to have a unit two point function. They are related to the operators introduced in (2.3) as follows:

$$
\begin{aligned}
& O_{R, r}^{\vec{m}, \vec{p}}(\sigma) \\
& \quad=\sqrt{\prod_{i=1}^{q} m_{i i}(\sigma) ! m_{i i}(\sigma) ! \prod_{k, l, k \neq l} m_{k \rightarrow l}(\sigma) ! p_{k \rightarrow l}(\sigma) ! \hat{O}_{R, r}^{\vec{m}, \vec{p}}(\sigma) .}
\end{aligned}
$$

Introduce the vectors $\left(v^{(i)}\right)_{a}=\delta_{i a}$ which form a basis for $V_{q}$. The vector $|\vec{p}, \vec{m}\rangle$ is defined as follows,

$$
|\vec{p}, \vec{m}\rangle=|\vec{p}\rangle \otimes|\vec{m}\rangle
$$

where

$$
\begin{aligned}
& |\vec{p}\rangle=\left(v^{(1)}\right)^{\otimes p_{1}} \otimes \cdots \otimes\left(v^{(q)}\right)^{\otimes p_{q}} \\
& |\vec{m}\rangle=\left(v^{(1)}\right)^{\otimes m_{1}} \otimes \cdots \otimes\left(v^{(q)}\right)^{\otimes m_{q}} .
\end{aligned}
$$

We will now explain how the sums over $\psi_{1}$ and $\psi_{2}$ in (2.18) can be evaluated. This discussion is novel and is one of the new contributions of this paper. Consider the term

$$
\begin{aligned}
T_{1}= & \sum_{\psi_{1} \in S_{\vec{p}} \times S_{\vec{m}}} \sum_{\psi_{2} \in S_{\vec{p}^{\prime}} \times S_{\vec{m}^{\prime}}}\left\langle\vec{p}^{\prime}, \vec{m}^{\prime}\left|\sigma_{2} \psi_{2}^{-1} E_{k i}^{(1)} \psi_{1}\right| \vec{p}, \vec{m}\right\rangle \\
& \times\left\langle\vec{p}, \vec{m}\left|\sigma_{1}^{-1} \psi_{1}^{-1} E_{i k}^{(p+1)} \psi_{2}\right| \vec{p}^{\prime}, \vec{m}^{\prime}\right\rangle .
\end{aligned}
$$

The dependence on the permutations $\sigma_{1}$ and $\sigma_{2}$ can be simplified with the following change of variables: replace $\psi_{2}$ with $\tilde{\psi}_{2}$, where

$$
\tilde{\psi}_{2}=\psi_{2} \sigma_{2}^{-1} \Rightarrow \tilde{\psi}_{2}^{-1}=\sigma_{2} \psi_{2}^{-1} .
$$

After relabeling $\tilde{\psi}_{2} \rightarrow \psi_{2}$ and taking the transpose of the first factor which is a real number, we find

$$
\begin{aligned}
T_{1}= & \sum_{\psi_{1} \in S_{\vec{p}} \times S_{\vec{m}}} \sum_{\psi_{2} \in S_{\vec{p}^{\prime}} \times S_{\vec{m}^{\prime}}}\left\langle\vec{p}, \vec{m}\left|\psi_{1}^{-1} E_{i k}^{(1)} \psi_{2}\right| \vec{p}^{\prime}, \vec{m}^{\prime}\right\rangle \\
& \times\left\langle\vec{p}, \vec{m}\left|\sigma_{1}^{-1} \psi_{1}^{-1} E_{i k}^{(p+1)} \psi_{2} \sigma_{2}\right| \vec{p}^{\prime}, \vec{m}^{\prime}\right\rangle .
\end{aligned}
$$

If $i \neq k$, the matrix element $\left\langle\vec{p}, \vec{m}\left|\psi_{1}^{-1} E_{i k}^{(1)} \psi_{2}\right| \vec{p}^{\prime}, \vec{m}^{\prime}\right\rangle$ is only nonvanishing if $\vec{p} \neq \vec{p}^{\prime}$ and $\vec{m}=\vec{m}^{\prime}$, while the matrix element $\left\langle\vec{p}, \vec{m}\left|\sigma_{1}^{-1} \psi_{1}^{-1} E_{i k}^{(p+1)} \psi_{2} \sigma_{2}\right| \vec{p}^{\prime}, \vec{m}^{\prime}\right\rangle$ is only nonvanishing if $\vec{p}=\vec{p}^{\prime}$ and $\vec{m} \neq \vec{m}^{\prime}$. Thus, $T_{1}$ vanishes for $i \neq k$. Indicate this explicitly as follows:

$$
\begin{aligned}
T_{1}= & \delta_{i k} \sum_{\psi_{1}, \psi_{2} \in S_{\vec{p}} \times S_{\vec{m}}}\left\langle\vec{p}, \vec{m}\left|\psi_{1}^{-1} E_{i i}^{(1)} \psi_{2}\right| \vec{p}, \vec{m}\right\rangle \\
& \times\left\langle\vec{p}, \vec{m}\left|\sigma_{1}^{-1} \psi_{1}^{-1} E_{i i}^{(p+1)} \psi_{2} \sigma_{2}\right| \vec{p}, \vec{m}\right\rangle .
\end{aligned}
$$

To simplify this expression further, note that $E_{i i}^{(1)}|\vec{p}, \vec{m}\rangle$ is only nonzero if vector $v^{(i)}$ occupies slot 1 in the vector $|\vec{p}\rangle$. In this case, $E_{i i}^{(1)}|\vec{p}, \vec{m}\rangle=|\vec{p}, \vec{m}\rangle$. Since $\psi_{1}$ and $\psi_{2}$ shuffle the vectors in $|\vec{p}, \vec{m}\rangle$ into all possible locations, $E_{i i}^{(1)}$ will in the end count how many times the vector $v^{(i)}$ appears in $|\vec{p}, \vec{m}\rangle$. This is given by $p_{i}$ introduced above. A similar argument applies to $E^{(p+1)}|\vec{p}, \vec{m}\rangle$. Thus, we obtain

$$
\begin{aligned}
T_{1}= & \delta_{i k} \frac{p_{i}}{p} \frac{m_{i}}{m} \sum_{\psi_{1}, \psi_{2} \in S_{\vec{p}} \times S_{\vec{m}}}\left\langle\vec{p}, \vec{m}\left|\psi_{1}^{-1} \psi_{2}\right| \vec{p}, \vec{m}\right\rangle \\
& \times\left\langle\vec{p}, \vec{m}\left|\sigma_{1}^{-1} \psi_{1}^{-1} \psi_{2} \sigma_{2}\right| \vec{p}, \vec{m}\right\rangle \\
= & \delta_{i k} \frac{p_{i}}{p} \frac{m_{i}}{m} \sum_{\psi_{1}, \psi_{2} \in S_{\vec{p}} \times S_{\vec{m}}} \sum_{h_{1}, h_{2} \in H_{X} \times H_{Y}} \delta\left(\psi_{1}^{-1} \psi_{2} h_{1}\right) \\
& \times \delta\left(\sigma_{1}^{-1} \psi_{1}^{-1} \psi_{2} \sigma_{2} h_{2}\right) .
\end{aligned}
$$

Now, perform the change of summation variables $\psi_{1} \rightarrow \tilde{\psi}_{1}$ with

$$
\psi_{1}=\psi_{2} \tilde{\psi}_{1}
$$

The summand is now independent of $\psi_{2}$ so that after summing over $\psi_{2}$ and relabeling $\tilde{\psi}_{1} \rightarrow \psi_{1}$ we find

$$
\begin{aligned}
T_{1}= & \delta_{i k}(p-1) !(m-1) ! p_{i} m_{i} \\
& \times \sum_{\psi_{1} \in S_{\vec{p}} \times S_{\vec{m}}} \sum_{h_{1}, h_{2} \in H_{X} \times H_{Y}} \delta\left(\psi_{1} h_{1}\right) \delta\left(\sigma_{1}^{-1} \psi_{1} \sigma_{2} h_{2}\right) .
\end{aligned}
$$

Summing over $\psi_{1}$ now gives 
$T_{1}=\delta_{i k}(p-1) !(m-1) ! p_{i} m_{i} \sum_{h_{1}, h_{2} \in H_{X} \times H_{Y}} \delta\left(\sigma_{1}^{-1} h_{1}^{-1} \sigma_{2} h_{2}\right)$.

We also need to consider the term

$$
\begin{aligned}
T_{4}= & \sum_{\psi_{1} \in S_{\vec{p}} \times S_{\vec{m}}} \sum_{\psi_{2} \in S_{\vec{p}^{\prime}} \times S_{\vec{m}^{\prime}}}\left\langle\vec{p}^{\prime}, \vec{m}^{\prime}\left|\sigma_{2} \psi_{2}^{-1} E_{k i}^{(p+1)} \psi_{1}\right| \vec{p}, \vec{m}\right\rangle \\
& \times\left\langle\vec{p}, \vec{m}\left|\sigma_{1}^{-1} \psi_{1}^{-1} E_{i k}^{(1)} \psi_{2}\right| \vec{p}^{\prime}, \vec{m}^{\prime}\right\rangle \\
= & \sum_{\psi_{1} \in S_{\vec{p}} \times S_{\vec{m}}} \sum_{\psi_{2} \in S_{\vec{p}^{\prime}} \times S_{\vec{m}^{\prime}}}\left\langle\vec{p}, \vec{m}\left|\sigma_{1}^{-1} \psi_{1}^{-1} E_{i k}^{(1)} \psi_{2}\right| \vec{p}^{\prime}, \vec{m}^{\prime}\right\rangle \\
& \times\left\langle\vec{p}, \vec{m}\left|\psi_{1}^{-1} E_{i k}^{(p+1)} \psi_{2} \sigma_{2}^{-1}\right| \vec{p}^{\prime}, \vec{m}^{\prime}\right\rangle .
\end{aligned}
$$

Changing variables $\psi_{1}^{-1} \rightarrow \sigma_{1}^{-1} \psi_{1}^{-1}$ shows that $T_{4}=T_{1}$ and hence

$$
\begin{aligned}
T_{1}+T_{4}= & 2 \delta_{i k}(p-1) !(m-1) ! p_{i} m_{i} \\
& \times \sum_{h_{1}, h_{2} \in H_{X} \times H_{Y}} \delta\left(\sigma_{1}^{-1} h_{1}^{-1} \sigma_{2} h_{2}\right) .
\end{aligned}
$$

The next sum we consider is

$$
\begin{aligned}
T_{2}= & \sum_{\psi_{1} \in S_{\vec{p}} \times S_{\vec{m}}} \sum_{\psi_{2} \in S_{\vec{p}^{\prime}} \times S_{\vec{m}^{\prime}}}\left\langle\vec{p}^{\prime}, \vec{m}^{\prime}\left|\sigma_{2} \psi_{2}^{-1} E_{c i}^{(1)} E_{k c}^{(p+1)} \psi_{1}\right| \vec{p}, \vec{m}\right\rangle \\
& \times\left\langle\vec{p}, \vec{m}\left|\sigma_{1}^{-1} \psi_{1}^{-1} E_{a k}^{(1)} E_{i a}^{(p+1)} \psi_{2}\right| \vec{p}^{\prime}, \vec{m}^{\prime}\right\rangle .
\end{aligned}
$$

Changing variables $\psi_{2}^{-1} \rightarrow \tilde{\psi}_{2}^{-1}$ with

$$
\tilde{\psi}_{2}^{-1}=\sigma_{2} \psi_{2}^{-1} \Rightarrow \tilde{\psi}_{2}=\psi_{2} \sigma_{2}^{-1},
$$

the sum becomes

$$
\begin{aligned}
T_{2}= & \sum_{\psi_{1} \in S_{\vec{p}} \times S_{\vec{m}}} \sum_{\psi_{2} \in S_{\vec{p}^{\prime}} \times S_{\vec{m}^{\prime}}}\left\langle\vec{p}^{\prime}, \vec{m}^{\prime}\left|\psi_{2}^{-1} E_{c i}^{(1)} E_{k c}^{(p+1)} \psi_{1}\right| \vec{p}, \vec{m}\right\rangle \\
& \times\left\langle\vec{p}, \vec{m}\left|\sigma_{1}^{-1} \psi_{1}^{-1} E_{a k}^{(1)} E_{i a}^{(p+1)} \psi_{2} \sigma_{2}\right| \vec{p}^{\prime}, \vec{m}^{\prime}\right\rangle \\
= & \sum_{\psi_{1} \in S_{\vec{p}} \times S_{\vec{m}}} \sum_{\psi_{2} \in S_{\vec{p}^{\prime}} \times S_{\vec{m}^{\prime}}}\left\langle\vec{p}^{\prime}, \vec{m}^{\prime}\left|\psi_{2}^{-1} \psi_{1} E_{c i}^{\psi_{1}^{-1}(1)} E_{k c}^{\psi_{1}^{-1}(p+1)}\right| \vec{p}, \vec{m}\right\rangle \\
& \times\left\langle\vec{p}, \vec{m}\left|\sigma_{1}^{-1} E_{a k}^{\psi_{1}^{-1}(1)} E_{i a}^{\psi_{1}^{-1}(p+1)} \psi_{1}^{-1} \psi_{2} \sigma_{2}\right| \vec{p}^{\prime}, \vec{m}^{\prime}\right\rangle .
\end{aligned}
$$

Change variables $\psi_{2} \rightarrow \rho$ with $\rho=\psi_{1}^{-1} \psi_{2}$, and relabel $\rho \rightarrow \psi_{2}$ to find

$$
\begin{aligned}
T_{2}= & \sum_{\psi_{1} \in S_{\vec{p}} \times S_{\vec{m}}} \sum_{\psi_{2} \in S_{\vec{p}^{\prime}} \times S_{\vec{m}^{\prime}}}\left\langle\vec{p}^{\prime}, \vec{m}^{\prime}\left|\psi_{2}^{-1} E_{c i}^{\psi_{1}^{-1}(1)} E_{k c}^{\psi_{1}^{-1}(p+1)}\right| \vec{p}, \vec{m}\right\rangle \\
& \times\left\langle\vec{p}, \vec{m}\left|\sigma_{1}^{-1} E_{a k}^{\psi_{1}^{-1}(1)} E_{i a}^{\psi_{1}^{-1}(p+1)} \psi_{2} \sigma_{2}\right| \vec{p}^{\prime}, \vec{m}^{\prime}\right\rangle .
\end{aligned}
$$

We will use $\hat{b}$ to denote the $q$-dimensional vector that has all entries zero except the $b$ th entry, which is 1 . For a nonzero contribution, the first factor requires

$$
\vec{p}-\hat{i}+\hat{c}=\vec{p}^{\prime} \quad \vec{m}-\vec{c}+\vec{k}=\vec{m}^{\prime}
$$

and the second factor requires

$$
\vec{m}-\hat{i}+\hat{a}=\vec{m}^{\prime} \quad \vec{p}-\vec{a}+\vec{k}=\vec{p}^{\prime}
$$

There are two solutions:

Case 1: $\hat{c}=\hat{i}$ and $\hat{a}=\hat{k}$. In this case $\vec{p}=\vec{p}^{\prime}$ and $\vec{m}-\hat{i}+\hat{k}=\vec{m}^{\prime}$,

Case 2: $\hat{c}=\hat{k}$ and $\hat{a}=\hat{i}$. In this case $\vec{m}=\vec{m}^{\prime}$ and $\vec{p}-\hat{i}+\hat{k}=\vec{p}^{\prime}$.

For case 1,

$$
\begin{aligned}
T_{2}= & \sum_{\psi_{1} \in S_{\vec{p}} \times S_{\vec{m}}} \sum_{\psi_{2} \in S_{\vec{p}^{\prime}} \times S_{\vec{m}^{\prime}}}\left\langle\vec{p}^{\prime}, \vec{m}^{\prime}\left|\psi_{2}^{-1} E_{i i}^{\psi_{1}^{-1}(1)} E_{k i}^{\psi_{1}^{-1}(p+1)}\right| \vec{p}, \vec{m}\right\rangle \\
& \times\left\langle\vec{p}, \vec{m}\left|\sigma_{1}^{-1} E_{k k}^{\psi_{1}^{-1}(1)} E_{i k}^{\psi_{1}^{-1}(p+1)} \psi_{2} \sigma_{2}\right| \vec{p}^{\prime}, \vec{m}^{\prime}\right\rangle .
\end{aligned}
$$

Consider the sum over $\psi_{1}$. Due to the factor $E_{k i}^{\psi_{1}^{-1}(p+1)}$, we get a nonzero contribution from the slots $p+1$, $p+2, \ldots, p+m$ (a $Y$ string) if a string starts from node $k$ and ends at node $i$. Thus, the sum over $\psi_{1}$ gives

$$
\begin{aligned}
T_{2} & =(p-1) !(m-1) ! p_{i \rightarrow k} m_{i i} \sum_{\psi_{2} \in S_{\vec{p} \times S_{\vec{m}^{\prime}}}}\left\langle\vec{p}, \vec{m}^{\prime}\left|\psi_{2}^{-1}\right| \vec{p}, \vec{m}^{\prime}\right\rangle\left\langle\vec{p}, \vec{m}^{\prime}\left|\sigma_{1}^{-1} \psi_{2} \sigma_{2}\right| \vec{p}, \vec{m}^{\prime}\right\rangle \\
& =(p-1) !(m-1) ! p_{i \rightarrow k} m_{i i} \sum_{\psi_{2} \in S_{\vec{p}} \times S_{\vec{m}^{\prime}}} \sum_{h_{1}, h_{2} \in H_{X} \times H_{Y}} \delta\left(\psi_{2}^{-1} h_{1}\right) \delta\left(\sigma_{1}^{-1} \psi_{2} \sigma_{2} h_{2}\right) \\
& =(p-1) !(m-1) ! p_{i \rightarrow k} m_{i i} \sum_{h_{1}, h_{2} \in H_{X} \times H_{Y}} \delta\left(\sigma_{1}^{-1} h_{1} \sigma_{2} h_{2}\right) .
\end{aligned}
$$


For case 2,

$$
T_{2}=\sum_{\psi_{1} \in S_{\vec{p}} \times S_{\vec{m}}} \sum_{\psi_{2} \in S_{\vec{p}^{\prime}} \times S_{\vec{m}}}\left\langle\vec{p}^{\prime}, \vec{m}\left|\psi_{2}^{-1} E_{k i}^{\psi_{1}^{-1}(1)} E_{k k}^{\psi_{k}^{-1}(p+1)}\right| \vec{p}, \vec{m}\right\rangle\left\langle\vec{p}, \vec{m}\left|\sigma_{1}^{-1} E_{i k}^{\psi_{1}^{-1}(1)} E_{i i}^{\psi_{i}^{-1}(p+1)} \psi_{2} \sigma_{2}\right| \vec{p}^{\prime}, \vec{m}\right\rangle .
$$

Consider the sum over $\psi_{1}$. We get a nonzero contribution for each $Y$ string starting from node $k$, which ends at node $i$. After summing over $\psi_{1}$, we have

$$
\begin{aligned}
T_{2} & =(p-1) !(m-1) ! p_{i i} m_{k \rightarrow i} \sum_{\psi_{2} \in S_{\vec{p}^{\prime}} \times S_{\vec{m}}}\left\langle\vec{p}^{\prime}, \vec{m}\left|\psi_{2}^{-1}\right| \vec{p}, \vec{m}^{\prime}\right\rangle\left\langle\vec{p}^{\prime}, \vec{m}\left|\sigma_{1}^{-1} \psi_{2} \sigma_{2}\right| \vec{p}^{\prime}, \vec{m}\right\rangle \\
& =(p-1) !(m-1) ! p_{i i} m_{k \rightarrow i} \sum_{\psi_{2} \in S_{\vec{p}^{\prime}} \times S_{\vec{m}}} \sum_{h_{1}, h_{2} \in H_{X} \times H_{Y}} \delta\left(\psi_{2}^{-1} h_{1}\right) \delta\left(\sigma_{1}^{-1} \psi_{2} \sigma_{2} h_{2}\right) \\
& =(p-1) !(m-1) ! p_{i i} m_{k \rightarrow i} \sum_{h_{1}, h_{2} \in H_{X} \times H_{Y}} \delta\left(\sigma_{1}^{-1} h_{1} \sigma_{2} h_{2}\right) .
\end{aligned}
$$

Armed with these sums, we now obtain a rather explicit expression for the matrix elements of $D_{2}^{X Y}$ in the Gauss graph basis:

$$
M_{R, r, \sigma_{1} T, t, \sigma_{2}}^{\vec{m}, \vec{p}}=-g_{\mathrm{YM}}^{2} \frac{\delta_{r u}}{\sqrt{\left|O_{R, r}^{\vec{m}, \vec{p}}\left(\sigma_{1}\right)\right|^{2}\left|O_{T, t}^{\vec{m}, \vec{p}}\left(\sigma_{2}\right)\right|^{2}}} \sum_{R^{\prime}} \delta_{R_{i}^{\prime} T_{k}^{\prime}} \sqrt{\frac{c_{R R^{\prime}} c_{T T^{\prime}}}{l_{R_{i}} l_{T_{k}}}}\left[2 \delta_{i k} p_{i} m_{i}-p_{k i} m_{i i}-p_{i i} m_{i k}\right] \sum_{h_{1}, h_{2} \in H_{X} \times H_{Y}} \delta\left(\sigma_{1}^{-1} h_{1} \sigma_{2} h_{2}\right) .
$$

This is the key result of this section and one of the key results of this paper. We will now describe how the above matrix can be diagonalized.

\section{BOSON LATTICE}

Our goal in this section is to diagonalize (2.31). This is achieved by interpreting (2.31) as the matrix elements of a Hamiltonian for bosons on a lattice. Toward this end, first note that the matrix elements $M_{R, r, \sigma_{1} T, t, \sigma_{2}}^{\vec{m}, \vec{p}}$ are only nonzero if we can choose coset representatives such that $\sigma_{1}$ and $\sigma_{2}$ describe the same element of $S_{m} \times S_{p}$. This implies that the brane-string systems described by $\sigma_{1}$ and $\sigma_{2}$ differ only in the number of strings with both ends attached to the same brane, but not in the number of string stretching between distinct branes. This already implies that the contribution $D_{2}^{X Y}$ only mixes eigenstates of $D_{2}^{X Z}$ and $D_{2}^{Y Z}$ that are degenerate and hence that all three are simultaneously diagonalizable. In this case, the matrix element in (2.31) simplifies to

$$
\begin{aligned}
& M_{R, r, \sigma_{1} T, t, \sigma_{2}}^{\vec{m}, \vec{p}} \\
& =-g_{\mathrm{YM}}^{2} \sqrt{\frac{\left|O_{R, r}^{\vec{m}, \vec{p}}\left(\sigma_{1}\right)\right|^{2}}{\left|O_{T, t}^{\vec{m}, \vec{p}}\left(\sigma_{2}\right)\right|^{2}}} \delta_{r u} \delta_{R_{i}^{\prime} T_{k}^{\prime}} \\
& \quad \times \sqrt{\frac{\left(N+l_{R_{i}}\right)\left(N+l_{T_{k}}\right)}{l_{R_{i}} l_{T_{k}}}} \\
& \quad \times\left[2 \delta_{i k} p_{i}\left(\sigma_{2}\right) m_{i}\left(\sigma_{2}\right)-p_{k i} m_{i i}\left(\sigma_{2}\right)-p_{i i}\left(\sigma_{2}\right) m_{i k}\right] .
\end{aligned}
$$

The number of strings stretching between the branes $m_{i k}$ (for $Y$ strings) and $p_{k i}$ (for $X$ strings) is the same for both systems, so

$$
m_{i k}\left(\sigma_{1}\right)=m_{i k}\left(\sigma_{2}\right) \equiv m_{i k} \quad p_{i k}\left(\sigma_{1}\right)=p_{i k}\left(\sigma_{2}\right) \equiv p_{i k} .
$$

It is the number of closed loops ( $m_{i i}$ for $Y$ loops and $p_{i i}$ for $X$ loops) that can differ between the operators that mix. Finally, we have introduced the notation

$$
p_{i}(\sigma)=\sum_{k \neq i} p_{i k}+p_{i i}(\sigma) \quad m_{i}(\sigma)=\sum_{k \neq i} m_{i k}+m_{i i}(\sigma) .
$$

From the structure of the operator mixing problem, we would expect that $M_{R, r, \sigma_{1} T, t, \sigma_{2}}^{\vec{m}, \vec{p}}=M_{T, t, \sigma_{2} R, r, \sigma_{1}}^{\vec{m}, \vec{m}}$. This is indeed the case, as a consequence of the easily checked identity

$$
\begin{aligned}
& \sqrt{\frac{\left|O_{R, r}^{\vec{m}, \vec{p}}\left(\sigma_{1}\right)\right|^{2}}{\left|O_{T, t}^{\vec{m}, \vec{p}}\left(\sigma_{2}\right)\right|^{2}}} \\
& \quad \times\left[2 \delta_{i k} p_{i}\left(\sigma_{2}\right) m_{i}\left(\sigma_{2}\right)-p_{k i} m_{i i}\left(\sigma_{2}\right)-p_{i i}\left(\sigma_{2}\right) m_{i k}\right] \\
& =\sqrt{\frac{\left|O_{T, t}^{\vec{m}, \vec{p}}\left(\sigma_{2}\right)\right|^{2}}{\left|O_{R, r}^{\vec{m}, \vec{p}}\left(\sigma_{1}\right)\right|^{2}}} \\
& \quad \times\left[2 \delta_{i k} p_{i}\left(\sigma_{1}\right) m_{i}\left(\sigma_{1}\right)-p_{k i} m_{i i}\left(\sigma_{1}\right)-p_{i i}\left(\sigma_{1}\right) m_{i k}\right],
\end{aligned}
$$

which holds for any $i$ and $k$.

The lattice model consists of two distinct species of bosons, one for $X$ and one for $Y$, hopping on a lattice, with a 
site for every brane or, equivalently, a site for every row in the Young diagram $R$ labeling the Gauss graph operator $\hat{O}_{R, r}^{\vec{m}, \vec{p}}(\sigma)$. The bosons are described by the following commuting sets of operators:

$$
\begin{array}{ll}
{\left[a_{i}, a_{j}^{\dagger}\right]=\delta_{i j} \quad\left[a_{i}^{\dagger}, a_{j}^{\dagger}\right]=0=\left[a_{i}, a_{j}\right]} \\
{\left[b_{i}, b_{j}^{\dagger}\right]=\delta_{i j}} & {\left[b_{i}^{\dagger}, b_{j}^{\dagger}\right]=0=\left[b_{i}, b_{j}\right] .}
\end{array}
$$

Using these boson oscillators, we have

$$
\begin{gathered}
m_{i i}=a_{i}^{\dagger} a_{i} \quad p_{i i}=b_{i}^{\dagger} b_{i} \\
m_{i}=\sum_{k} m_{i k}+a_{i}^{\dagger} a_{i} \quad p_{i}=\sum_{k} p_{i k}+b_{i}^{\dagger} b_{i} .
\end{gathered}
$$

The vacuum of the Fock space $|0\rangle$ obeys

$$
a_{i}|0\rangle=0=b_{i}|0\rangle \quad i=1,2, \ldots, q .
$$

The Hamiltonian of the lattice model is given by

$$
\begin{aligned}
H= & \sum_{i, j=1}^{q} \sqrt{\frac{\left(N+l_{R_{i}}\right)\left(N+l_{R_{j}}\right)}{l_{R_{i}} l_{R_{j}}}}\left(2 \delta_{i j}\left(\sum_{l \neq i} p_{i l}+b_{i}^{\dagger} b_{i}\right)\right. \\
& \left.\times\left(\sum_{l \neq i} m_{i l}+a_{i}^{\dagger} a_{i}\right)-p_{j i} a_{j}^{\dagger} a_{i}-m_{j i} b_{j}^{\dagger} b_{i}\right) .
\end{aligned}
$$

Notice that this Hamiltonian is quadratic in each type of oscillator. It has a nontrivial repulsive interaction given by the $\sum_{i} a_{i}^{\dagger} a_{i} b_{i}^{\dagger} b_{i}$ term, which makes it energetically unfavorable for $a$ and $b$ type particles to sit on the same site. Also, the full Fock space is a tensor product between the Fock space for the $a$ oscillator and the Fock space for the $b$ oscillator. We will use the occupation number representation to describe the boson states. To complete the mapping to the lattice model, we need to explain the correspondence between Gauss graph operators and states of the boson lattice. This map is given by reading the boson occupation numbers for each site from the number of closed strings with both ends attached to the node corresponding to that site. In the next subsection, we consider an example which nicely illustrates this map.

Finally, let us make an important observation regarding (3.9). Although the eigenvalues of this Hamiltonian are subleading contributions to the anomalous dimension, there is an important situation in which this correction is highly significant: for BPS states, the leading contribution to the anomalous dimension vanishes, and this subleading correction is important. The BPS operators are labeled by Gauss graphs that have $p_{i k}=m_{i k}=0$ whenever $i \neq k$; i.e., there are no strings stretching between branes. In this case, it is clear that (3.9) vanishes, so the BPS operators remain BPS when the subleading interactions are included.

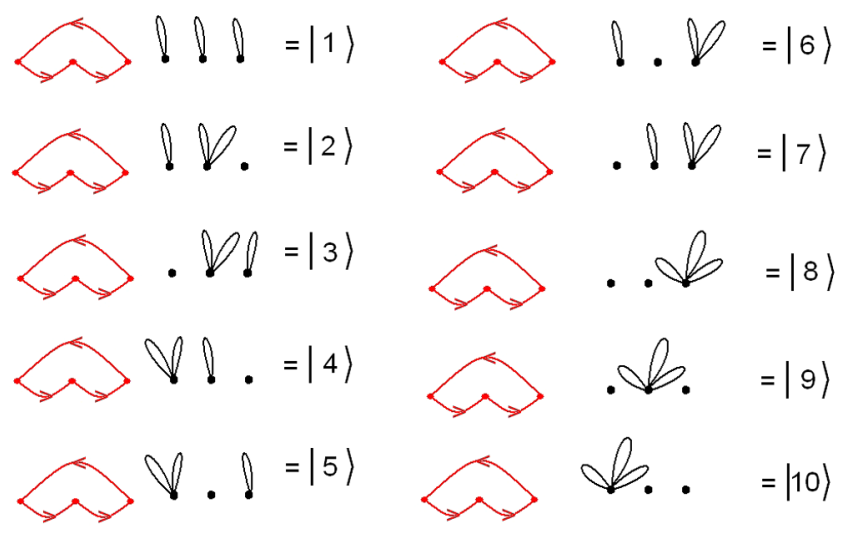

FIG. 2. Each Gauss graph label is composed of two graphs, the first for the $X$ strings and the second for the $Y$ strings. Each graph has three nodes (because $q=3$ ). There are no $b$ type particles because there are no closed $X$ strings. There are three $a$ type particles because there are three closed $Y$ strings. All operators share the same $r$ label.

\section{A. Example}

In this section, we will consider an example for which $R$ has $q=3$ rows and $p=m=3$. In this problem, ten operators mix. The Gauss graph labels for the operators that mix are displayed in Fig. 2.

For the Gauss graph operators shown, we have the following correspondence with boson lattice states:

$$
\begin{aligned}
|1\rangle & =a_{1}^{\dagger} a_{2}^{\dagger} a_{3}^{\dagger}|0\rangle & |2\rangle & =a_{1}^{\dagger} \frac{\left(a_{2}^{\dagger}\right)^{2}}{\sqrt{2 !}}|0\rangle \\
|3\rangle & =a_{3}^{\dagger} \frac{\left(a_{2}^{\dagger}\right)^{2}}{\sqrt{2 !}}|0\rangle & |4\rangle & =a_{2}^{\dagger} \frac{\left(a_{1}^{\dagger}\right)^{2}}{\sqrt{2 !}}|0\rangle \\
|5\rangle & =a_{3}^{\dagger} \frac{\left(a_{1}^{\dagger}\right)^{2}}{\sqrt{2 !}}|0\rangle & |6\rangle & =a_{1}^{\dagger} \frac{\left(a_{3}^{\dagger}\right)^{2}}{\sqrt{2 !}}|0\rangle \\
|7\rangle & =a_{2}^{\dagger} \frac{\left(a_{3}^{\dagger}\right)^{2}}{\sqrt{2 !}}|0\rangle & |8\rangle & =\frac{\left(a_{3}^{\dagger}\right)^{3}}{\sqrt{3 !}}|0\rangle \\
|9\rangle & =\frac{\left(a_{2}^{\dagger}\right)^{3}}{\sqrt{3 !}}|0\rangle & |10\rangle & =\frac{\left(a_{1}^{\dagger}\right)^{3}}{\sqrt{3 !}}|0\rangle .
\end{aligned}
$$

It is now rather straightforward to compute matrix elements of the lattice Hamiltonian. For example,

$$
\langle 1|H| 2\rangle=-\sqrt{\frac{\left(N+l_{R_{3}}\right)\left(N+l_{R_{2}}\right)}{l_{R_{2}} l_{R_{3}}}} \sqrt{2} .
$$

It is instructive to compare this to the answer coming from (2.31). To move from state 2 to state 1 , a string must detach from node 2 and reattach to node 3 . Thus, we should plug $i=2$ and $k=1$ into (2.31). The Gauss graph $\sigma_{1}$ corresponds to $|1\rangle$, while $\sigma_{2}$ corresponds to $|2\rangle$. In addition, $R_{2}^{\prime}=T_{1}^{\prime}$, and from the Gauss graphs, we read off $p_{32}=1$ 

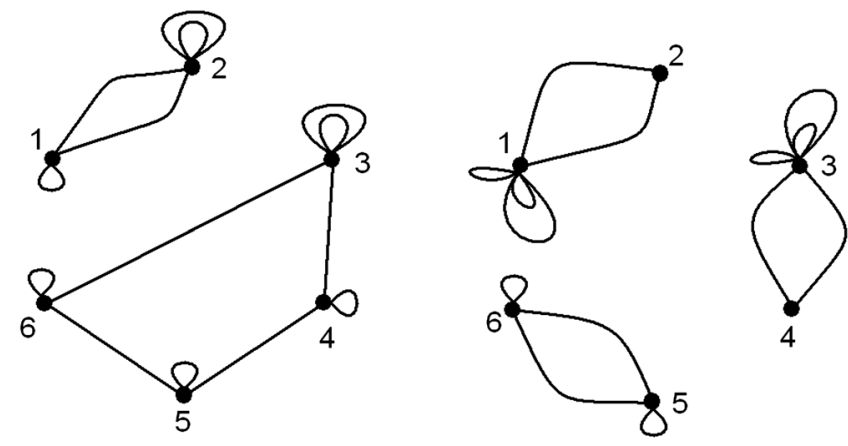

FIG. 3. An example of a Gauss graph with nonzero $a$ and $b$ occupation numbers.

and $m_{22}\left(\sigma_{1}\right)=1$. It is now simple to see that (2.31) is in complete agreement with the above matrix element.

Finally, the state corresponding to the Gauss graph in Fig. 3 is

$$
\frac{\left(a_{1}^{\dagger}\right)^{3}}{\sqrt{3 !}} \frac{\left(a_{3}^{\dagger}\right)^{3}}{\sqrt{3 !}} a_{5}^{\dagger} a_{6}^{\dagger} b_{1}^{\dagger} \frac{\left(b_{2}^{\dagger}\right)^{2}}{\sqrt{2 !}} \frac{\left(b_{3}^{\dagger}\right)^{2}}{\sqrt{2 !}} b_{4}^{\dagger} b_{5}^{\dagger} b_{6}^{\dagger}|0\rangle .
$$

\section{DIAGONALIZATION}

In this section, we will consider a class of examples that can be diagonalized explicitly. Our main motivation is to show that working with the lattice is simple, so the mapping we have found is useful.

\section{A. Exact eigenstates}

For these examples, take

$$
p_{k i}=p_{i k}=\delta_{k, i+1} B \quad m_{k i}=m_{i k}=\delta_{k, i+1} A,
$$

with $A$ and $B$ two positive integers. For examples of Gauss graphs that obey this condition, see Fig. 4. There are two cases we will consider: we will fix the number of $a$ particles to zero and leave the number of $b$ particles arbitrary or fix the number of $b$ particles to zero and leave the number of $a$ particles arbitrary. We will also specialize to labels $R$ that have the difference between any two row lengths
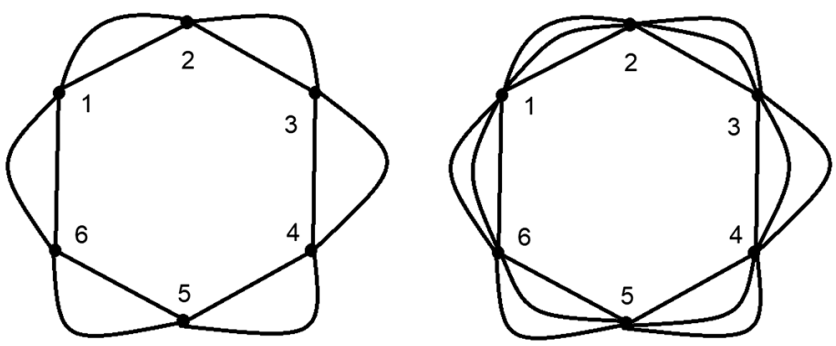

FIG. 4. An example of a Gauss graph that is easily solvable. The example shown has $A=2$ and $B=3$.
$l_{R_{i}}-l_{R_{j}} \sim N$, but $\frac{l_{R_{i}}-l_{R_{j}}}{l_{R_{i}}} \approx 0$. In this case, our lattice Hamiltonian simplifies to

$$
\begin{aligned}
H= & \frac{\left(N+l_{R_{1}}\right)}{l_{R_{1}}} \sum_{i=1}^{q}\left(2\left(B+b_{i}^{\dagger} b_{i}\right)\left(A+a_{i}^{\dagger} a_{i}\right)\right. \\
& \left.-B\left(a_{i}^{\dagger} a_{i+1}+a_{i+1}^{\dagger} a_{i}\right)-A\left(b_{i}^{\dagger} b_{i+1}+b_{i+1}^{\dagger} b_{i}\right)\right) .
\end{aligned}
$$

This Hamiltonian is easily diagonalized by going to Fourier space. Indeed, in terms of the new oscillators,

$$
\begin{aligned}
\tilde{a}_{n} & =\frac{1}{\sqrt{q}} \sum_{k=1}^{q} e^{i \frac{2 \pi k n}{q}} a_{k} \quad \tilde{b}_{n}=\frac{1}{\sqrt{q}} \sum_{k=1}^{q} e^{i \frac{2 \pi k n}{q}} b_{k} \\
n & =0,1, \ldots, q-1,
\end{aligned}
$$

the Hamiltonian becomes (we have set the number of $a$ particles to zero)

$$
\begin{aligned}
H= & A \frac{\left(N+l_{R_{1}}\right)}{l_{R_{1}}} \sum_{n=0}^{q-1}\left(2-2 \cos \left(\frac{2 \pi n}{q}\right)\right) \tilde{b}_{n}^{\dagger} \tilde{b}_{n} \\
& +2 A B q \frac{\left(N+l_{R_{1}}\right)}{l_{R_{1}}} .
\end{aligned}
$$

Eigenstates of the lattice Hamiltonian are given by arbitrary momentum space excitations

$$
\prod_{n=0}^{q-1} \frac{\left(\tilde{a}_{n}^{\dagger}\right)^{\alpha_{n}}}{\sqrt{\alpha_{n} !}}|0\rangle \quad \text { or } \quad \prod_{n=0}^{q-1} \frac{\left(\tilde{b}_{n}^{\dagger}\right)^{\beta_{n}}}{\sqrt{\beta_{n} !}}|0\rangle,
$$

where the occupation numbers $\alpha_{n}$ and $\beta_{n}$ are arbitrary. This state can be translated back into the Gauss graph language to give operators of a definite scaling dimension.

\section{B. General properties of low energy eigenstates}

In this section, we will sketch the features of generic low energy states of the lattice Hamiltonian. We begin by relaxing the constraint that only one species is hopping. In the end, we will also make comments valid for the general Gauss graph configuration. The Hamiltonian becomes

$$
\begin{gathered}
H=H_{a}+H_{b}+H_{a b}+E_{0} \\
H_{a}=\frac{\left(N+l_{R_{1}}\right)}{l_{R_{1}}} B \sum_{i=1}^{q}\left(2 a_{i}^{\dagger} a_{i}-a_{i}^{\dagger} a_{i+1}-a_{i+1}^{\dagger} a_{i}\right) \\
H_{b}=\frac{\left(N+l_{R_{1}}\right)}{l_{R_{1}}} A \sum_{i=1}^{q}\left(2 b_{i}^{\dagger} b_{i}-b_{i}^{\dagger} b_{i+1}-b_{i+1}^{\dagger} b_{i}\right) \\
H_{a b}=\frac{\left(N+l_{R_{1}}\right)}{l_{R_{1}}} \sum_{i=1}^{q} 2 b_{i}^{\dagger} b_{i} a_{i}^{\dagger} a_{i} .
\end{gathered}
$$


The constant $E_{0}=2 A B q \frac{\left(N+l_{R_{1}}\right)}{l_{R_{1}}}$ is not important for the dynamics but must be included to obtain the correct anomalous dimensions. To start, consider $H_{a}$, which is a kinetic term for the $a$ particles. The first term in the Hamiltonian implies that it costs energy to have an $a$ particle occupying a site, while the second and third terms tell us this energy can be lowered by hopping between sites $i$ and $i+1$. Consequently, to minimize $H_{a}$, the $a$ particles will spread out as much as is possible. This is in perfect accord with the results of the last section. The lowest energy single particle state is the zero momentum state, which occupies each site with the same probability: the particle spreads out as much as is possible. Very similar reasoning for $H_{b}$ implies that the $b$ particles will also spread out as much as is possible. Finally, the term $H_{a b}$ is a repulsive interaction, telling us that it costs energy to have $a$ 's and $b$ 's occupying the same site. So, there is a competition going on: the terms $H_{a}$ and $H_{b}$ want to spread the $a$ 's and $b$ 's uniformly on the lattice, which would certainly distribute $a$ 's and $b$ 's to the same site. The term $H_{a b}$ wants to ensure that any particular site will have only $a$ 's or $b$ 's but not both. Which wins?

Consider a thermodynamiclike limit where we consider a very large number of both species of particles, $n_{a}$ and $n_{b}$. In the end, the low energy state will be a "demixed" state with no sites holding both $a$ 's and $b$ 's. To see this, note that $H_{a}$ grows like $n_{a}$ and $H_{b}$ grows like $n_{b}$. This is much smaller than the growth of the term $H_{a b}$, which grows like $n_{a} n_{b}$, so the repulsive interaction wins. This conclusion is nicely borne out by numerical results for the two-component BoseHubbard model [36,37]. The ground state phase diagram of the Hamiltonian of Ref. [36] shows four distinct phases: the double superfluid phase, supercounterflow phase, demixed Mott insulator phase, and demixed superfluid phase. Comparing our Hamiltonian to that of Ref. [36], we are always in the demixed superfluid phase; the $a$ and $b$ particles do not mix but are free to move in their respective domains. Thus, we have a collection of two species of particles that demix but are free on their respective domains. It is in this sense that we have an essentially free system.

For the generic Gauss graph, with any choices for the values of $m_{i k}$ and $p_{i k}$, it is clear that $H_{a}$ and $H_{b}$ will still cause the $a$ and $b$ particles to spread out as much as possible. The term $H_{a b}$ will again dominate when we have large numbers of $a$ 's and $b$ 's, so we again expect a demixed gas. We can translate this structure of the generic state back into the language of the giant graviton description. Up to now, we have considered dual giant gravitons which correspond to operators labeled by Young diagrams with long rows. Recall that dual giant gravitons wrap an $\mathrm{S}^{3} \subset \mathrm{AdS}_{5}$. In this context, $l_{R_{1}}$ is the momentum of each giant, and $N+l_{R_{1}}$ is the radius on the LLM plane at which the giant orbits. The Hamiltonian for giant gravitons, which wrap $S^{3} \subset S^{5}$, is given by

$$
\begin{aligned}
H= & \frac{\left(N-l_{R_{1}}\right)}{l_{R_{1}}} \sum_{i=1}^{q}\left(2\left(B+b_{i}^{\dagger} b_{i}\right)\left(A+a_{i}^{\dagger} a_{i}\right)\right. \\
& \left.-B\left(a_{i}^{\dagger} a_{i+1}+a_{i+1}^{\dagger} a_{i}\right)-A\left(b_{i}^{\dagger} b_{i+1}+b_{i+1}^{\dagger} b_{i}\right)\right) .
\end{aligned}
$$

These operators are labeled by Young diagrams with long columns. The giants orbit on the LLM plane with a radius of $N-l_{R_{1}}$. The $X$ and $Y$ fields are each charged under different $U(1)$ s of the $\mathcal{R}$-symmetry group. The $\mathcal{R}$ symmetry of the CFT translates into angular momentum of the dual string theory, so attaching the particles to a given giant corresponds to giving the giant angular momentum. The lowest energy giant graviton states are obtained by distributing the momenta carried by the $X$ and $Y$ fields evenly between the giants with the condition that any particular giant carries only $X$ or $Y$ momenta, but not both. These conclusions hold for the generic state where there are enough $p_{i k}$ and $m_{i k}$ nonzero, allowing the $X$ 's and $Y$ 's to hop between any two giants, possibly by a complicated path. Thus, in the end, we see that the mapping to the boson lattice model has allowed a rather detailed understanding of the operator mixing problem.

\section{CONCLUSIONS}

In this article, we have studied the action of the one-loop dilatation operator $D_{2}$ on Gauss graph operators $O_{R, r}^{\vec{m}, \vec{p}}(\sigma)$ which belong to the $S U(3)$ sector. The term we have studied, $D_{2}^{X Y}$, is diagonal in the $r$ label, mixing operators labeled by distinct graphs. It makes a subleading contribution as compared to $D_{2}^{X Z}$ and $D_{2}^{Y Z}$ when $n \gg m+p$. The two leading terms mix operators labeled by distinct $r \mathrm{~s}$. Diagonalizing the action of $D_{2}^{X Z}$ and $D_{2}^{Y Z}$ on $r$ leads to a collection of decoupled harmonic oscillators, which we refer to as the $Z$ oscillators, since the $r$ label is associated with $Z$. The spectrum of the $Z$ oscillators gives the leading contribution to the anomalous dimensions. The new contribution that we have studied in this paper can also be mapped to a collection of oscillators, describing a lattice boson model. This is done by introducing two sets of oscillators, the $X$ and $Y$ oscillators associated to the $X$ and $Y$ fields. Diagonalizing the $X$ and $Y$ oscillators breaks degeneracies among different copies of $Z$ oscillators and leads to a constant addition to their ground state energy. This is then a constant shift of the anomalous dimension. Although this shift is subleading (it is of order $\frac{m}{n}$ ), it could potentially show that certain states are not in fact BPS. This was investigated in detail, and it turns out that states that are BPS (their leading order anomalous dimension vanishes) at leading order remain BPS when the subleading correction is computed (it, too, vanishes).

The mapping that we have found to a lattice boson model has achieved an enormous simplification of the operator mixing problem, and we have managed to understand it in 
some detail. Indeed, using the lattice boson model, we have argued that the lowest energy giant graviton states are obtained by distributing the momenta carried by the $X$ and $Y$ fields evenly between the giants with the condition that any particular giant carries only $X$ or $Y$ momenta, but not both. Since states with two charges are typically $\frac{1}{4}$-BPS while states with three charges are typically $\frac{1}{8}$-BPS, it maybe that the solution is locally trying to maximize supersymmetry. It would be interesting to arrive at the same picture, employing the dual string theory description.

Perhaps the most interesting consequence of our results is that they suggest ways in which one can go beyond the $\frac{1}{2}$ BPS sector. Indeed, all three types of fields considered have been mapped to oscillators, so perhaps there is a more general description of this sector that treats all three types of oscillators on the same footing. This would relax the constraint $n \gg p+m$ which allows for operators that are far from the $\frac{1}{2}$-BPS limit. Deriving this picture is a fascinating open problem, since it will require that we go beyond the displaced corners approximation or, alternatively, that we generalize it.

As a final comment, recall that Mikhailov [38] has constructed an infinite family of $\frac{1}{8} \mathrm{BPS}$ giant graviton branes in $\mathrm{AdS}_{5} \times \mathrm{S}^{5}$. Quantizing the space of Mikhailov's solutions leads to $N$ noninteracting bosons in a harmonic oscillator [39-41]. It is tempting to speculate that it is precisely these oscillators that we are uncovering in our study; for evidence in harmony with this suggestion, see Ref. [42]. It would be interesting to make this speculation precise.

\section{ACKNOWLEDGMENTS}

We thank Vishnu Jejjala, Sanjaye Ramgoolam, and Izak Snyman for helpful discussions. This work is supported by the South African Research Chairs Initiative of the Department of Science and Technology and National Research Foundation as well as funds received from the National Institute for Theoretical Physics.
[1] J. M. Maldacena, The large N limit of superconformal field theories and supergravity, Int. J. Theor. Phys. 38, 1113 (1999); , The large $\mathrm{N}$ limit of superconformal field theories and supergravity, Adv. Theor. Math. Phys. 2, 231 (1998).

[2] S. S. Gubser, I. R. Klebanov, and A. M. Polyakov, Gauge theory correlators from noncritical string theory, Phys. Lett. B 428, 105 (1998).

[3] E. Witten, Anti-de Sitter space and holography, Adv. Theor. Math. Phys. 2, 253 (1998).

[4] N. Gromov, V. Kazakov, S. Leurent, and D. Volin, Quantum Spectral Curve for Planar $\mathcal{N}=4$ Super-Yang-Mills Theory, Phys. Rev. Lett. 112, 011602 (2014).

[5] J. A. Minahan and K. Zarembo, The Bethe ansatz for $N=4$ superYang-Mills, J. High Energy Phys. 03 (2003) 013.

[6] N. Beisert et al., Review of AdS/CFT integrability: An overview, Lett. Math. Phys. 99, 3 (2012).

[7] N. Beisert, V. A. Kazakov, K. Sakai, and K. Zarembo, The algebraic curve of classical superstrings on $\mathrm{AdS}_{5} \times S_{5}$, Commun. Math. Phys. 263, 659 (2006).

[8] H. Lin, O. Lunin, and J. M. Maldacena, Bubbling AdS space and 1/2 BPS geometries, J. High Energy Phys. 10 (2004) 025 .

[9] J. McGreevy, L. Susskind, and N. Toumbas, Invasion of the giant gravitons from Anti-de Sitter space, J. High Energy Phys. 06 (2000) 008.

[10] M. T. Grisaru, R. C. Myers, and O. Tafjord, SUSY and goliath, J. High Energy Phys. 08 (2000) 040.

[11] A. Hashimoto, S. Hirano, and N. Itzhaki, Large branes in AdS and their field theory dual, J. High Energy Phys. 08 (2000) 051.
[12] V. Balasubramanian, M. Berkooz, A. Naqvi, and M. J. Strassler, Giant gravitons in conformal field theory, J. High Energy Phys. 04 (2002) 034.

[13] D. Berenstein, Shape and holography: Studies of dual operators to giant gravitons, Nucl. Phys. B675, 179 (2003).

[14] S. Corley, A. Jevicki, and S. Ramgoolam, Exact correlators of giant gravitons from dual $N=4$ SYM theory, Adv. Theor. Math. Phys. 5, 809 (2001).

[15] R. de Mello Koch, J. Smolic, and M. Smolic, Giant gravitons-with strings attached (I), J. High Energy Phys. 06 (2007) 074.

[16] Y. Kimura and S. Ramgoolam, Branes, anti-branes and brauer algebras in gauge-gravity duality, J. High Energy Phys. 11 (2007) 078.

[17] T. W. Brown, P. J. Heslop, and S. Ramgoolam, Diagonal multi-matrix correlators and BPS operators in $N=4$ SYM, J. High Energy Phys. 02 (2008) 030.

[18] R. Bhattacharyya, S. Collins, and R. de Mello Koch, Exact multi-matrix correlators, J. High Energy Phys. 03 (2008) 044.

[19] T. W. Brown, P. J. Heslop, and S. Ramgoolam, Diagonal free field matrix correlators, global symmetries and giant gravitons, J. High Energy Phys. 04 (2009) 089.

[20] Y. Kimura and S. Ramgoolam, Enhanced symmetries of gauge theory and resolving the spectrum of local operators, Phys. Rev. D 78, 126003 (2008).

[21] Y. Kimura, Correlation functions and representation bases in free $N=4$ Super Yang-Mills, Nucl. Phys. B865, 568 (2012).

[22] R. de Mello Koch, J. Smolic, and M. Smolic, Giant gravitons-with Strings Attached (II), J. High Energy Phys. 09 (2007) 049. 
[23] D. Bekker, R. de Mello Koch, and M. Stephanou, Giant gravitons-with strings attached. III., J. High Energy Phys. 02 (2008) 029.

[24] T. W. Brown, Permutations and the loop, J. High Energy Phys. 06 (2008) 008.

[25] R. de Mello Koch, G. Mashile, and N. Park, Emergent threebrane lattices, Phys. Rev. D 81, 106009 (2010).

[26] V. De Comarmond, R. de Mello Koch, and K. Jefferies, Surprisingly simple spectra, J. High Energy Phys. 02 (2011) 006.

[27] W. Carlson, R. de Mello Koch, and H. Lin, Nonplanar integrability, J. High Energy Phys. 03 (2011) 105.

[28] R. de Mello Koch, M. Dessein, D. Giataganas, and C. Mathwin, Giant graviton oscillators, J. High Energy Phys. 10 (2011) 009.

[29] R. de Mello Koch, G. Kemp, and S. Smith, From large N nonplanar anomalous dimensions to open spring theory, Phys. Lett. B 711, 398 (2012).

[30] R. de Mello Koch and S. Ramgoolam, A double coset ansatz for integrability in AdS/CFT, J. High Energy Phys. 06 (2012) 083.

[31] R. de Mello Koch, N. H. Tahiridimbisoa, and C. Mathwin, Anomalous dimensions of heavy operators from magnon energies, J. High Energy Phys. 03 (2016) 156.

[32] R. de Mello Koch, C. Mathwin, and H. J. R. van Zyl, LLM magnons, J. High Energy Phys. 03 (2016) 110.
[33] N. Beisert, The SU(2/2) dynamic S-matrix, Adv. Theor. Math. Phys. 12, 948 (2008).

[34] R. de Mello Koch, S. Graham, and W. Mabanga, Subleading corrections to the Double Coset Ansatz preserve integrability, J. High Energy Phys. 02 (2014) 079.

[35] V. Balasubramanian, D. Berenstein, B. Feng, and M.-x. Huang, D-branes in Yang-Mills theory and emergent gauge symmetry, J. High Energy Phys. 03 (2005) 006.

[36] F. Lingua, M. Guglielmino, V. Penna, and B. C. Sansone, Demixing effects in mixtures of two bosonic species, Phys. Rev. A 92, 053610 (2015).

[37] T. Mishra, R. V. Pai, and B. P. Das, Phase separation in a two-species Bose mixture, Phys. Rev. A 76, 013604 (2007).

[38] A. Mikhailov, Giant gravitons from holomorphic surfaces, J. High Energy Phys. 11 (2000) 027.

[39] C. E. Beasley, BPS branes from baryons, J. High Energy Phys. 11 (2002) 015.

[40] I. Biswas, D. Gaiotto, S. Lahiri, and S. Minwalla, Supersymmetric states of $N=4$ Yang-Mills from giant gravitons, J. High Energy Phys. 12 (2007) 006.

[41] G. Mandal and N. V. Suryanarayana, Counting 1/8-BPS dual-giants, J. High Energy Phys. 03 (2007) 031.

[42] R. de Mello Koch and L. Nkumane, From gauss graphs to giants, J. High Energy Phys. 02 (2018) 005. 